\title{
The Consequences of Endogenizing Information for the Performance of a Sequential Decision Procedure*
}

(Forthcoming Journal of Economic Behavior and Organization)

\author{
Otto H. Swank ${ }^{\dagger}$ \\ Erasmus University Rotterdam and Tinbergen Institute \\ Bauke Visser \\ Erasmus University Rotterdam and Tinbergen Institute
}

May 8, 2006

\begin{abstract}
We analyze the implications of endogenizing information collection and reputational concerns for the performance of a sequential decision structure. In this model, two agents decide in a sequence whether or not to implement a public project. The cost of gathering information is private. We derive two results. First, endogenising information replaces the herding problem by a free-rider problem. Second, endogenising information aggravates the distortionary effect of reputational concerns.
\end{abstract}

*A previous version of this paper was entitled "The Consequences of Endogenizing Information for Herd Behavior". We are grateful to two anonymous referees, an associate editor and Alan Kirman for their constructive comments

${ }^{\dagger}$ swank@few.eur.nl

†bvisser@few.eur.nl 


\section{Introduction}

In the last three decades, several studies have appeared on the relationship between the various ways decisions are made in organizations on the one hand and the performance of organizations on the other hand. A seminal paper is by Sah and Stiglitz (1986), who studied situations in which individuals have to screen projects. ${ }^{1}$ In their article, the way the screening process is organised is important for two reasons. First, individuals make errors of judgement. The implication of this aspect is that it is not always optimal to delegate the screening decision to one individual. Second, there are costs of acquiring and communicating information. The more individuals are involved in the screening process, the higher are these costs.

It is not very surprising that in a Sah and Stiglitz setting, sequential decision procedures are quite attractive. To understand why, consider an organizational unit responsible for the screening of project proposals the organization receives. One could think of, say, drafts of books submitted for publication with a publisher, music demos sent to a record company, or a request for a loan filed with a bank. Suppose the unit is made up of two persons. The expected benefits are negative so that implementation of the project requires that both individuals accept the project. Suppose a sequential decision procedure in which the project is first evaluated by one person. If this person accepts, then the project is forwarded to the second person who makes the final decision. If the first person rejects, then the project is discarded. ${ }^{2}$ Clearly, compared to a situation in which two persons simultaneously evaluate projects, the sequential decision procedure saves on evaluation costs. If the first person rejects the projects, the second person does not have to evaluate the project anymore. Compared to a situation in which the decision is made by a single individual, the sequential decision procedure has the advantage that some errors of judgement of the first person can be corrected by the second person.

Without doubt, the literature building on Sah and Stiglitz has yielded many insights into the pros and cons of alternative decision procedures. However, a main

\footnotetext{
${ }^{1}$ Related papers include Sah and Stiglitz (1988), Young (1988), Ladha (1992), Koh (1992), BenYashar and Nitzan (1997), Gehrig et al. (2000), and Visser (2000). Other seminal contributions include Marschak and Radner (1972) and Keren and Levhari (1983).

${ }^{2}$ In case the expected benefits of the project are positive so that status quo requires that two individuals reject the project, then the decision is forwarded to the second individual if and only if the first individual rejects.
} 
drawback is that it ignores the effect of the internal structure of an organization on its members' incentives to acquire information.

In this paper we examine the performance of a standard two-step sequential decision procedure in a situation in which the individuals participating do not automatically behave in line with the interest of the organization. Our model differs from that of Sah and Stiglitz in two important aspects. First, we endogenise the process of opinion formation or information acquisition. ${ }^{3}$ To see the relevance of this deviation, consider the examples of the selection processes given above. Evaluating the merits of a book or a demo, and the risks associated with a potential borrower takes time and effort. Moreover, whether effort is exerted is a matter of choice. Each individual has to decide whether or not to collect information. Given that in Sah and Stiglitz the cost of information collection plays an essential role, endogenising the acquisition of information seems to be a natural extension of their model.

The second deviation is that we assume that apart from caring about the project payoff, individuals are concerned with their reputation as decision makers. Arguably, one of the key assets of a publisher, a record company, or a bank is its ability to separate the wheat from the chaff. It is therefore likely that people who are successful at identifying good proposals are worth more to the organization and are therefore more likely to be kept and promoted. As a consequence, an employee evaluating the quality of a proposal is likely to care about his perceived screening ability. We assume that there are two types of employees, smart and dumb ones. Smart employees are more likely to make correct decisions than dumb employees. The reputation of an individual is defined as the (posterior) probability that he is smart.

We derive two results. First, if reputational concerns are sufficiently strong and the individuals' abilities do not differ too much, then the first individual in the decision process does not collect information and always delegates the decision about the project to the second individual. Evidently, the performance of a sequential decision procedure is then identical to the performance of a procedure that delegates the decision about the project immediately to agent 2. To understand our first result, suppose that the first individual in the decision process collected information and accepted the project. As we know from the herding literature, reputational concerns

\footnotetext{
${ }^{3}$ For another recent study that pays attention to information acquisition in a Sah and Stiglitz setting see Gehrig (2004) and the comments by Demougin (2004).
} 
give then an incentive to the second individual to accept the project too, irrespective of her information. As information is endogenous, agent 2 therefore decides not to exert effort to acquire information. Agent 1 anticipates the behaviour of agent 2 . The behaviour of agent 2, however, is conditional on the behaviour of agent 1 . By not collecting information, agent 1 eliminates agent 2's incentive to refrain from exerting effort. At this point, the public good feature of information is important. Concerning information collection, each agent wants to free-ride on the other agent. Agent 1 can do so by directly forwarding the decision to agent 2 .

Another way of putting our first result is that endogenising information in a model with reputational concerns changes the possible distortion in the decision process from herding to free-riding. Our second result is that endogenising information aggravates the distortion, in the sense that with endogenous information free-riding occurs for a wider range of parameters than herding with exogenous information.

The practical implication of our findings is that where a sequential decision structure would have been best from a project payoff perspective, in practice we may see that the decision is taken by a single person. The loafing makes it unattractive to keep two employees.

To illustrate the relevance of our paper, first consider the way a typical Dutch literary publisher evaluates a new manuscript submitted for publication. ${ }^{4}$ First, the publisher checks whether or not the manuscript fits in its collection. Next, the real screening starts. The book is given to an editor. Only if this editor has a positive view, another editor (or an external reader) is involved. If this agent is also positive, the author will be invited for a meeting.

Now consider the way a typical Dutch bank evaluates a request for a standard loan (up to euro 250,000). ${ }^{5}$ In the first stage, a commercial agent helps the client to submit a proposal. Once the proposal has been completed, it is sent to a financial analyst who evaluates its merits. If the proposal is approved, it is checked whether the analyst has the authority to approve the proposal. If so, the loan can be provided.

The difference between the publisher case and the bank case is clear. The pub-

\footnotetext{
${ }^{4}$ See the website set up by nine Dutch publishers at http://www.boekboek.nl/boekboek/show/id=2808.

${ }^{5}$ Rabobank Group (internal document for provision of loans, and personal communication with J.G. Schuitemaker).
} 
lisher case is a typical example of a sequential decision procedure as discussed at the beginning of the introduction. In the bank case, the screening of standard loans is done by one agent. After the screening, the procedure is checked (in particular, whether the analyst has the authority to approve the proposal). Our paper gives a possible explanantion for the difference between the two screening procedures. This explanantion requires that relative to financial analysts, editors care more about their product, and less about their reputation.

Apart from the literature on the internal structure of organizations, our paper is related to the literature on herd behaviour. Our model is similar in spirit to one studied by Scharfstein and Stein (1990). They consider a situation in which agents make decisions with a view to manipulating inferences regarding their ability. Like us, they consider a situation in which agents make decisions sequentially. Our model differs from Scharfstein and Stein in three respects. First, in our model, the agents make a decision about one public project instead of two private projects. Second, information is endogenised. Third, agents do not only care about their reputation but are also concerned with project outcomes. ${ }^{6}$ One of the main results of Scharfstein and Stein is that the second agent has an incentive to mimic the first agent, by ignoring private information. This incentive also plays an essential role in our model. With endogenous information and a public project, however, the incentive to mimic leads to free-riding rather than herding.

Our paper is also closely related to Ottaviani and Sorensen (2001) who study the consequences of statistical and reputational herding for the optimal order in which agents speak. They show that reputational concerns may imply that it is better to let agents participate in the decision process who are not too smart. As we will show, this also holds true in our model. In their model, however, information is exogenous and agents are exclusively concerned with their reputation.

The remainder of this paper is organised as follows. The next section sets out the model. Section 3 analyses the model and presents the equilibria. Section 4 analyzes a model in which agents attach a direct cost to disagreeing with each other. Section 5 summarises our main results. Proofs can be found in the appendix.

\footnotetext{
${ }^{6}$ Scharfstein and Stein (1990) briefly discusses this extension.
} 


\section{The Model}

\subsection{Decision Procedure}

Two agents $i=1,2$ have to make a decision about a project. This decision is made sequentially. Agent 1 is the first to look at the project. If he decides to reject the project, $X_{1}=0$, the status quo is maintained. If instead he decides to accept the project, $X_{1}=1$, it moves on to the second agent, whose verdict is final. That is, project implementation requires $\left\{X_{1}=1, X_{2}=1\right\}$, whereas $\left\{X_{1}=0\right\}$ and $\left\{X_{1}=1, X_{2}=0\right\}$ imply that the status quo will be maintained.

There are two states of the world $\mu \in\{-h, h\}$. Each state occurs with an ex ante probability $\frac{1}{2}$. If the project is implemented, the project yields $p+\mu$ utility to each agent, where $p$ denotes the expected benefit of the project. By normalisation, status quo does not deliver utility. Throughout, we assume that $-h<p<0$. The implication of this assumption is that the correct decision about the project depends on the state of the world. Moreover, without further information about $\mu$, each agent prefers rejection to implementation.

Before deciding on the project, an agent $i$ may examine the pros and cons of the project. The effort that comes with this is costly to the agent, $C$. Whether effort is of any use depends on the type $t_{i}$ or quality of agent $i$ who undertakes the research. A smart agent, $t=s m$, is more likely to benefit from the outcomes than a dumb agent. In fact, we assume that a smart agent fully benefits from the research he undertakes: the signal $s_{i}=\{b, g\}$ he receives is fully informative $\left(s_{i}=g\right.$ if and only if $\mu=h ; s_{i}=b$ if and only if $\mu=-h$ ). Any research undertaken by a dumb agent, $t=d u$, on the other hand, is to no avail. That is, a dumb agent receives an uninformative signal: $s_{i}=g$ with probability $\frac{1}{2}$, independent of the true state of the world ${ }^{7}$. If no effort is exerted, the agent bases his decision on his prior knowledge and his position in the decision structure. We assume that agents do not know whether they are smart or dumb. Agent $i$ only knows that he is smart with probability $\pi_{i}$. This probability is common knowledge.

Let us briefly justify why we deviate from the Sah and Stiglitz setting by assuming that agents have different types. In Sah and Stiglitz, agents do not differ in

\footnotetext{
${ }^{7}$ Qualitatively the same results would be obtained if one were to assume that a smart (dumb) agent receives an informative signal with probability $\eta_{s}\left(\eta_{d}\right)$, with $\eta_{s}>\eta_{d}$.
} 
types, but signals differ in quality. The reason for assuming types of agents rather than types of signals is our focus on reputational concerns. In our model, agents are concerned about how the market perceives their abilities. These concerns lead to herding with exogenous information, and as we show in our model, to free riding with endogenous information. For reputational concerns to be effective, the existence of types of agents is required.

\subsection{Timing}

At the beginning of the game, nature chooses the state of the world, and the types of agents. Next, agent 1 decides whether to exert effort. Then in the absence or presence of a signal he must decide whether to reject the project, $X_{1}=0$, or to recommend implementation, $X_{1}=1$. We assume that an agent can show that he has not exerted effort. The motivation of this assumption is that information collection takes time. By choosing $X_{1}=1$ instantaneously, agent 1 can signal that he has not exerted effort. However, an agent cannot prove that he has exerted effort. Waiting does not "prove" that agent 1 has exerted effort. If agent 1 has chosen implementation, the project moves on for evaluation by agent 2. Agent 2 decides whether to exert effort or not, and whether the project should be implemented, $X_{2}=1$, or rejected, $X_{2}=0$. At the end of the game, the organization updates its beliefs about the probability that each agent is smart, based on the individual decisions made by the agents, $\left\{X_{1}=1\right\},\left\{X_{1}=1, X_{2}=1\right\}$, and $\left\{X_{1}=1, X_{2}=0\right\}$. We assume that the organization does not observe the true state of the world when

the project is implemented. At the end of this paper, we will come back to this assumption.

\subsection{Preferences}

The agents are concerned both with the outcomes of the project - the expected project payoff - and with their reputation. Agent $i$ 's reputation is defined as the posterior probability that $i$ is smart, $\widehat{\pi}_{i}$. The payoff to $i$, net of any cost of exerting 
effort $C$, equals

$$
\begin{aligned}
U_{i}\left(X_{1}=1, X_{2}=1\right) & =p+\mu+\lambda \widehat{\pi}_{i}\left(X_{1}=1, X_{2}=1\right) \\
U_{i}\left(X_{1}=1, X_{2}=0\right) & =\lambda \widehat{\pi}_{i}\left(X_{1}=1, X_{2}=0\right) \\
U_{i}\left(X_{1}=0\right) & =\lambda \widehat{\pi}_{i}\left(X_{1}=0\right)
\end{aligned}
$$

In equation (1), $\lambda$ denotes the weight an agent places on reputation relative to the outcomes of the project.

\subsection{The Economic Environment}

We make two sets of assumptions in order to guarantee an interesting economic environment. First, we assume that in case of decision making by a single agent, a situation we call delegation, this agent will exert effort and follow his signal. This amounts to assuming that ${ }^{8,9}$

Assumption 1 In case of single agent decision making ('delegation'), an agent implements the project only if the signal is positive, $p+\pi_{i} h>0$ for both $i=1,2$.

Assumption 2 In case of single agent decision making, an agent exerts effort, $\frac{1}{2}\left(p+\pi_{i} h\right)>C$ for both $i=1,2$.

Note that a bad signal leads to project rejection without further assumptions as $p-\pi_{i} h<p<0$. A similar set of assumptions is made to make a sequential decision structure interesting and viable if agents are only driven by the project's payoff. That is, conditional on having exerted effort, the agents should be willing to follow their respective signals. Assumption 1 ensures that two positive signals lead to implementation. What remains to be guaranteed is that in case of conflicting signals $s_{1} \neq s_{2}$ the expected project payoff net of costs of effort is negative or zero,

Assumption 3 In case of two conflicting signals, the project payoff is negative or zero, $p+E\left(\mu \mid s_{i}=g, s_{j}=b\right)=p+\frac{\pi_{i}-\pi_{j}}{1-\pi_{i} \pi_{j}} h \leq 0$

\footnotetext{
${ }^{8}$ Note that if the condition in Assumption 2 holds, so does the one in Assumption 1. We prefer to present these assumptions separately because they refer to different stages in the decision making process.

${ }^{9}$ In the Appendix, part A.2, we show that the mathematical statements imply the desired behaviour.
} 
Were this not the case, one would want to delegate the decision to agent $i$, as a good signal received by this agent would have such a large likelihood to be informative that the worst possible information the other agent can receive (a negative signal) would not change the verdict on the project. With assumption 3, a 'statistical cascade' will not occur as it is not rational to ignore a second, bad signal ${ }^{10}$. We further assume that if agent 1 exerts effort and follows his signal, the increase in expected project payoff thanks to the addition of agent 2's effort exceeds the costs of this additional effort. Conditional on agent 1 having exerted effort and following his signal, implementation by agent 2 without exerting effort yields $p+\pi_{1} h$. If agent 2 exerts effort and follows his signal his payoff becomes $\frac{1}{2}\left(1+\pi_{1} \pi_{2}\right) p+\frac{1}{2}\left(\pi_{1}+\pi_{2}\right) h-$ C.

Assumption 4 If agent 1 exerts effort and follows his signal, the increase in expected project payoff thanks to agent 2's effort exceeds the costs of this effort, $\frac{1}{2}\left(\pi_{1} \pi_{2}-1\right) p+$ $\frac{1}{2}\left(\pi_{2}-\pi_{1}\right) h>C$.

Similarly, without reputational concerns agent 1 would be willing to exert effort and follow his signal if agent 2 already exerts effort and follows her signal. This requires that

Assumption 5 If agent 2 exerts effort and follows his signal, agent 1's effort is efficient, $\frac{1}{4}\left(\pi_{1} \pi_{2}-1\right) p+\frac{1}{4}\left(\pi_{1}-\pi_{2}\right) h>C$.

The assumptions (3), (4) and (5) are necessary and sufficient to make a sequential decision structure viable and interesting. ${ }^{11}$

\section{Analysis}

Ours is a dynamic model with incomplete information. An equilibrium specifies for each agent $i$ a decision rule that determines whether to exert effort, and whether to accept the project - possibly conditional on a signal - such that expected utility is maximised, given the strategy of the other agent and the posterior beliefs held by

\footnotetext{
${ }^{10}$ For an introduction to the cascading literature, see Bikhchandani, Hirschleifer, and Welch (1998) and the references therein.

${ }^{11}$ Note once again that if assumptions (4) and (5) hold so does assumption (3).
} 
the market. For the market, it means that the posterior beliefs are formed according to Bayes' rule ${ }^{12}$.

\subsection{Agent 2}

We start by analysing the behaviour of agent 2 who has received a project from agent 1 . Suppose agent 1 exerted effort and followed his signal. In this section we analyse the best reply of agent 2 in this situation. Although we assume that agent 2 cannot observe with what likelihood agent 1 exerted effort, in equilibrium this is known. In Section 3.2, we analyse under which conditions agent 1 exerts effort and show that if agent 1 exerts effort, he will always follow his signal.

\section{Posterior beliefs}

Below we will argue that if agent 2 receives a positive signal, she has no incentive to reject the project. However, if agent 2 receives a negative signal, she may vote for implementation. Therefore, let $\beta$ be the probability with which agent 2 exerts effort, let $\gamma$ be the probability the project is accepted if she receives a signal that is negative, and assume the project is accepted if she receives a positive signal. Lemma 1 states the posterior beliefs concerning agent 2's type.

Lemma 1 Suppose agent 1 exerts effort and follows his signal. Suppose $X_{2}=1$ if $s_{2}=g$. Let $\beta=\operatorname{Pr}(2$ exerts effort $)$ and $\gamma=\operatorname{Pr}\left(X_{2}=1 \mid s_{2}=b\right)$. Then the posterior beliefs about agent 2's type equal

$$
\begin{aligned}
& \widehat{\pi}_{2}\left(X_{1}=1, X_{2}=0\right)=\left(\frac{1-\pi_{1}}{1-\pi_{1} \pi_{2}}\right) \pi_{2}<\pi_{2} \\
& \widehat{\pi}_{2}\left(X_{1}=1, X_{2}=1\right)=\frac{2-\beta(1-\gamma)\left(1-\pi_{1}\right)}{2-\beta(1-\gamma)\left(1-\pi_{1} \pi_{2}\right)} \pi_{2} \geq \pi_{2}
\end{aligned}
$$

Observe that, once agent 1 has passed on the project for a final decision to agent 2 , the reputation of the latter is hurt by project rejection. The reason is that project rejection by agent 2 reveals that she received a negative signal, or, more importantly, a signal different from agent 1's. As smart people who exert effort receive identical signals, opposing signals reduce the probability that either agent, including the second agent, is smart. By the same token, agent 2 strengthens her

\footnotetext{
${ }^{12}$ Bayes' rule will be used where possible. A plausible posterior will be provided otherwise.
} 
reputation by mimicking agent 1's decision as this suggests she received a positive signal.

\section{Project Choice}

We continue to assume that agent 1 exerts effort and follows his signal. Assume agent 2 has exerted effort and has therefore received a signal. The question becomes what agent 2 decides on the project if $s_{2}=g$ and $s_{2}=b$. The main result of the herding literature applies. Given that both agents exert effort and thus possess private information, sufficiently strong reputational concerns induce agent 2 to ignore her private signal and to mimic agent 1 . In case of a positive signal, she prefers project implementation to rejection as this leads both to a positive project payoff and to a better reputation. In case of a negative signal, rejection gives her

$$
\lambda \widehat{\pi}_{2}\left(X_{1}=1, X_{2}=0\right)-C
$$

whereas implementation yields

$$
p+\frac{\pi_{1}-\pi_{2}}{1-\pi_{1} \pi_{2}} h+\lambda \widehat{\pi}_{2}\left(X_{1}=1, X_{2}=1\right)-C
$$

Therefore, if agent 2 received signal $s_{2}=b$, whether she prefers rejection to implementation depends on the sign of

$$
\left(p+\frac{\pi_{1}-\pi_{2}}{1-\pi_{1} \pi_{2}} h\right)+\lambda\left(\widehat{\pi}_{2}\left(X_{1}=1, X_{2}=1\right)-\widehat{\pi}_{2}\left(X_{1}=1, X_{2}=0\right)\right)
$$

The first term in brackets is negative by Assumption 3, p $\frac{\pi_{1}-\pi_{2}}{1-\pi_{1} \pi_{2}} h<0$. She ignores a negative signal and accepts the project, $\gamma=1$, if she cares considerably about her reputation, $\lambda>\lambda^{* *}$. The negative expected project payoff stemming from implementation is more than offset by the increase in ex post reputation. Only if she cares little about her reputation, $\lambda<\lambda^{*}$, will she refrain from accepting the project in case of a negative signal, $\gamma=0$. For intermediate values, $\lambda \in\left[\lambda^{*}, \lambda^{* *}\right]$, she sometimes ignores her negative signal and accepts the project, $\gamma \in[0,1] .{ }^{13}$

\footnotetext{
${ }^{13}$ Of course, the posterior belief $\widehat{\pi}_{2}\left(X_{1}=1, X_{2}=1\right)$ depends on and is consistent with each type of behaviour of agent 2 (i.e., with the value of $\gamma$ ), see the proof of Lemma 2
} 
Lemma 2 Suppose agent 1 exerts effort and follows his signal. Suppose agent 2 exerts effort. Then, for $\lambda<\lambda^{*}$, agent 2 follows her signal, $\gamma^{*}=0$; for $\lambda \in\left[\lambda^{*}, \lambda^{* *}\right]$, she sometimes ignores a bad signal ('probabilistic herding'), $\gamma^{*} \in[0,1]$; and for $\lambda>\lambda^{* *}$, she herds with probability one, $\gamma^{*}=1$.

In particular, if agent 2 cares exclusively about her reputation, which amounts to $\lambda \rightarrow \infty$ in our model, she always herds.

\section{Effort}

Now that we have derived the behaviour of agent 2 conditional on effort having been exerted, we turn to the conditions which rule her decision to exert effort or not. If agent 2 does not exert effort, $\beta=0$, it is optimal to implement the project. Recall that we have assumed that decision making by one agent yields more than always maintaining the status quo, see assumption 1. Essentially, not exerting effort amounts to delegating the decision to agent 1. Agent 2's payoff then equals:

$$
p+\pi_{1} h+\lambda \widehat{\pi}_{2}\left(X_{1}=1, X_{2}=1\right)
$$

If agent 2 exerts effort, she anticipates how she will react to signals. As shown in lemma 2 , if agent 2 cares considerably about her reputation, $\lambda>\lambda^{* *}$, she will herd if she exerts effort - a project that reaches her desk is implemented irrespective of her signal. Clearly, in this case, agent 2 does not exert effort and always implements a project that lands on her desk - nobody is willing to pay for information that will surely not be used.

If agent 2 cares to a moderate degree about her reputation, $\lambda \in\left[\lambda^{*}, \lambda^{* *}\right]$, exerting effort and sometimes implementing a project in case of $s_{2}=b$ yields a payoff equal to

$$
\begin{aligned}
& \frac{1}{2}\left(1+\pi_{1} \pi_{2}\right)\left(p+\frac{\pi_{1}+\pi_{2}}{1+\pi_{1} \pi_{2}} h+\lambda \widehat{\pi}_{2}\left(X_{1}=1, X_{2}=1\right)\right)+ \\
& \frac{1}{2}\left(1-\pi_{1} \pi_{2}\right)\left(p+\frac{\pi_{1}-\pi_{2}}{1-\pi_{1} \pi_{2}} h+\lambda \widehat{\pi}_{2}\left(X_{1}=1, X_{2}=1\right)\right)-C \\
= & p+\pi_{1} h+\lambda \widehat{\pi}_{2}\left(X_{1}=1, X_{2}=1\right)-C
\end{aligned}
$$

In deriving (8), we used that if agent 2 mixes in case of a bad signal $s_{2}=b$, a signal she receives with probability $\frac{1}{2}\left(1-\pi_{1} \pi_{2}\right)$, she is indifferent between implementation 
and rejection. This implies that before observing the signal $s_{2} \in\{g, b\}$, agent 2 knows that implementation yields at least as much as rejection. Under such circumstances, it does not make sense to exert costly effort to obtain a signal. This is also borne out by a comparison of equations (8) and (7). For $\lambda \in\left[\lambda^{*}, \lambda^{* *}\right]$, then, agent 2 prefers not exerting effort to exerting effort. Lemma 3 sums up the discussion so far.

Lemma 3 If information is costly, neither herding nor probabilistic herding occurs in equilibrium. Instead of (probabilistic) herding taking place, information is not acquired.

The situation that remains to be studied is the one in which agent 2 cares relatively little about her reputation, $\lambda<\lambda^{*}$. In that case, with the costs of effort sunk, information is used efficiently. In particular, a signal $s_{2}=b$ leads to project rejection as the gain in reputation stemming from project acceptance is more than offset by the reduction in project payoff. As without information agent 2 would accept the project, exerting effort is only useful if a negative signal is obtained and leads to a rejection of the project (a positive signal leads to project acceptance). For $\lambda$ less than but very close to $\lambda^{*}$, agent 2 would have followed her negative signal if she were to have it for free. However, for any positive costs, she is unwilling to exert the effort and incur the costs. In other words, for any cost level $C$, there is a value $\bar{\lambda}_{C}<\lambda^{*}$ such that for $\lambda>\bar{\lambda}_{C}$, agent 2 is unwilling to exert effort, even though she would use the information efficiently once it were there. The benefit of exerting effort and then following one's signal as compared with not exerting effort and implementing the project is the increase in project payoff. Exerting effort is however costly per se, and hurts one's reputation in the event of a negative signal. Only if agent 2 cares sufficiently little about her reputation, $\lambda<\underline{\lambda}_{C}$, where $\underline{\lambda}_{C}<\bar{\lambda}_{C}<\lambda^{*}$, will agent 2 follow her signal and exert effort. Lemma 4 states the exact conditions. ${ }^{14}$

Lemma 4 Suppose agent 1 exerts effort and follows his signal. Furthermore suppose that the posterior beliefs are given by (2) and (3). Then, the unique best reply of

\footnotetext{
${ }^{14}$ Note that we do not report behaviour of agent 2 in case she receives a bad signal, $\gamma$, if $\lambda>\bar{\lambda}_{C}$ as she refrains from exerting effort, $\beta=0$, and will never receive a signal in the first place. Of course, the values of $\underline{\lambda}_{C}$ and $\bar{\lambda}_{C}$ depend on and are consistent with the behaviour of agent 2 , see the proof.
} 
agent 2 is as follows. For $\lambda<\underline{\lambda}_{C}$, agent 2 exerts effort and follows her signal, $\beta^{*}=1, \gamma^{*}=0$. For $\lambda \in\left[\underline{\lambda}_{C}, \bar{\lambda}_{C}\right]$, agent 2 exerts effort with probability $\beta^{*}$, where $\beta^{*}$ is the root of (A.11), and follows her signal if she has one, $\gamma^{*}=0$. For $\lambda>\bar{\lambda}_{C}$, agent 2 exerts no effort, and chooses $X_{2}=1, \beta^{*}=0, \gamma^{*}=1$. Furthermore, $\underline{\lambda}_{C}<\bar{\lambda}_{C}<\lambda^{*}$.

By Assumption 2, if agent 1 does not exert effort - if he delegates the decision on the project to agent 2 - agent 2 is better off exerting effort and following her signal than following any other strategy.

\subsection{Agent 1 and Equilibrium Selection}

\section{Project Choice}

We now analyse the behaviour of agent 1 . We first assume he has exerted effort and received a signal. Suppose he has received a negative signal, $s_{1}=b$. The best he can do is to maintain the status quo. First, on the basis of Assumption 3, even if agent 2 were to receive a positive signal, expected project payoff would be negative. Second, passing on the project to agent 2 would not improve his expected reputation. Now suppose he has received a positive signal, $s_{1}=g$. Rejection would lead to a project payoff equal to zero. Acceptance either leads to agent 2 exerting effort and following her signal (for $\lambda<\underline{\lambda}_{C}$ ), or to agent 2 being indifferent between exerting effort or not (for $\lambda \in\left[\underline{\lambda}_{C}, \bar{\lambda}_{C}\right]$ ), or to agent 2 preferring not to exert effort and implement the project without further ado $\left(\lambda>\bar{\lambda}_{C}\right)$. In any case, expected project payoffs are larger than zero, while the expected reputation of agent 1 is left unaffected. Hence, acceptance dominates rejection in case of a good signal.

Lemma 5 In any equilibrium in which agent 1 exerts effort, he follows his signal. Effort

Now consider the effort decision. By assumption, agent 1 can show that he has not exerted effort. If agent 1 does not exert effort, he prefers delegating the decision about the project to rejecting it out of hand. His payoff equals

$$
\frac{1}{2}\left(p+\pi_{2} h\right)+\lambda \pi_{1}
$$


If agent 1 does exert effort, agent 2's reaction is described in Lemma 4. Three cases have to be distinguished. First, if agent 2 cares little about her reputation, $\lambda<\underline{\lambda}_{C}$, she exerts effort and follows her signal if agent 1 does. In subsection 2.4, we have characterized the economic environment in such a way that agent 1 is willing to exert effort if agent 2 exerts effort and follows her signal, see assumption 5 .

Lemma 6 For $\lambda<\underline{\lambda}_{C}$, agent 1 exerts effort (and follows his signal).

For the two remaining cases, $\lambda \in\left[\underline{\lambda}_{C}, \bar{\lambda}_{C}\right]$ and $\lambda>\bar{\lambda}_{C}$, it is important to observe that if agent 1 is the only agent to exert effort (because agent 2 does not exert effort and implements a project with probability one if agent 1 exerts effort), then the payoff for agent 1 equals

$$
\frac{1}{2}\left(p+\pi_{1} h\right)+\lambda \pi_{1}-C .
$$

A comparison of expressions (9) and (10) shows that if

$$
\frac{1}{2}\left(\pi_{1}-\pi_{2}\right) h \geq C
$$

agent 1 (weakly) prefers to decide about the project himself rather than letting the decision on the project depend solely on agent 2 . As a consequence, if this inequality holds, agent 1 prefers exerting effort to delegating the decision to agent 2 both if the latter agent cares to a moderate degree about her reputation, $\lambda \in\left[\underline{\lambda}_{C}, \bar{\lambda}_{C}\right]$, and if she cares considerably about her reputation, $\lambda>\bar{\lambda}_{C}$.

Consider first the case that $\lambda \in\left[\underline{\lambda}_{C}, \bar{\lambda}_{C}\right]$. Then from Lemma 4 we know that with probability $\beta^{*}$ agent 2 exerts effort and follows her signal if agent 1 exerts effort. We know by assumption 5 that agent 1 then benefits from exerting effort. With probability $1-\beta^{*}$ agent 2 does not exert effort but implements the project. If equation (11) holds, agent 1 still prefers to exert effort rather than delegating the decision to agent 2 . That is, whether agent 2 exerts effort or not, agent 1 prefers exerting effort to not exerting effort.

Similarly, for $\lambda>\bar{\lambda}_{C}$, agent 2 does not exert effort but implements the project if agent 1 exerts effort. Once again, if equation (11) holds, agent 1 prefers to exert effort rather than delegating the decision to agent 2. 
Lemma 7 For $\frac{1}{2}\left(\pi_{1}-\pi_{2}\right) h \geq C$, agent 1 exerts effort independent of the behaviour of agent 2. In particular, the degree to which agent 2 cares about his reputation is unimportant.

If instead

$$
\frac{1}{2}\left(\pi_{1}-\pi_{2}\right) h<C
$$

agent 1 prefers delegating the decision on the project to agent 2 compared to being the only one to decide on the project. For $\lambda>\bar{\lambda}_{C}$, then, agent 1 refrains from exerting effort and delegates the decision to agent 2. If equality (12) holds and $\lambda \in\left[\underline{\lambda}_{C}, \bar{\lambda}_{C}\right]$, agent 1's choice of exerting effort or not depends on the likelihood with which agent 2 exerts effort. If $\beta^{*}$ is close to one, there is a high likelihood that if agent 1 exerts effort so will agent 2 . The resulting payoff is larger than what agent 1 can obtain by not exerting effort himself. On the other hand, if $\beta^{*}$ is close to zero, it is very likely that agent 2 does not exert effort if agent 1 does. In this case, agent 1 would be better off by delegating the decision to agent 2 . There is therefore some value $\bar{\beta} \in(0,1)$ satisfying

$$
\begin{aligned}
& \bar{\beta}\left[\frac{1}{4}\left(1+\pi_{1} \pi_{2}\right) p+\frac{1}{4}\left(\pi_{1}+\pi_{2}\right) h+\lambda \pi_{1}-C\right]+ \\
& (1-\bar{\beta})\left[\frac{1}{2}\left(p+\pi_{1} h\right)+\lambda \pi_{1}-C\right]=\frac{1}{2}\left(p+\pi_{2} h\right)+\lambda \pi_{1}
\end{aligned}
$$

such that for $\beta^{*}>\bar{\beta}$, agent 1 exerts effort, whereas for $\beta^{*}<\bar{\beta}$ he does not exert effort but delegates the decision making to agent 2 . He is indifferent for $\beta^{*}=\bar{\beta}$ and we assume he does not exert effort. As agent 1 cannot influence the expected value of his ex post reputation, $\bar{\beta}$ is independent of $\lambda$.

Lemma 8 Assume $\frac{1}{2}\left(\pi_{1}-\pi_{2}\right) h<C$. If $\lambda>\bar{\lambda}_{C}$, then agent 1 exerts no effort. If $\lambda \in\left[\underline{\lambda}_{C}, \bar{\lambda}_{C}\right]$, then agent 1 exerts effort if and only if $\beta^{*}>\bar{\beta}$, where $\bar{\beta}$ solves (13) and $\beta^{*}$ is the root of $(A .11)$.

We are now ready to state the equilibrium of this game.

Proposition 1 The equilibrium strategies for agents 1 and 2 are as follows. 
1. If $\lambda<\underline{\lambda}_{C}$, both agents exert effort and follow their respective signals. Posterior beliefs equal $\widehat{\pi}_{2}\left(X_{1}=1, X_{2}=0\right)=\left(\frac{1-\pi_{1}}{1-\pi_{1} \pi_{2}}\right) \pi_{2}$ and $\widehat{\pi}_{2}\left(X_{1}=1, X_{2}=1\right)=$ $\left(\frac{1+\pi_{1}}{1+\pi_{1} \pi_{2}}\right) \pi_{2}$.

2. If $\lambda \in\left[\underline{\lambda}_{C}, \bar{\lambda}_{C}\right]$ and $\frac{1}{2}\left(\pi_{1}-\pi_{2}\right) h \geq C$, agent 1 exerts effort and follows his signal, whereas agent 2 exerts effort with probability $\beta^{*}$ and follows her signal if she has one, $\gamma^{*}=0$. Posterior beliefs equal $\widehat{\pi}_{2}\left(X_{1}=1, X_{2}=1\right)=$ $\frac{2-\beta\left(1-\pi_{1}\right)}{2-\beta\left(1-\pi_{1} \pi_{2}\right)} \pi_{2}$ and $\widehat{\pi}_{2}\left(X_{1}=1, X_{2}=0\right)=\left(\frac{1-\pi_{1}}{1-\pi_{1} \pi_{2}}\right) \pi_{2}$.

3. If $\lambda \in\left[\underline{\lambda}_{C}, \bar{\lambda}_{C}\right], \frac{1}{2}\left(\pi_{1}-\pi_{2}\right) h<C$ and $\beta^{*}>\bar{\beta}$, where $\beta^{*}$ is the root of (A.11), agent 1 exerts effort and follows his signal, and agent 2 exerts effort with probability $\beta^{*}$, and follows her signal if she has one, $\gamma^{*}=0$. Posterior beliefs equal $\widehat{\pi}_{2}\left(X_{1}=1, X_{2}=1\right)=\frac{2-\beta\left(1-\pi_{1}\right)}{2-\beta\left(1-\pi_{1} \pi_{2}\right)} \pi_{2}$ and $\widehat{\pi}_{2}\left(X_{1}=1, X_{2}=0\right)=$ $\left(\frac{1-\pi_{1}}{1-\pi_{1} \pi_{2}}\right) \pi_{2}$.

4. If $\lambda \in\left[\underline{\lambda}_{C}, \bar{\lambda}_{C}\right], \frac{1}{2}\left(\pi_{1}-\pi_{2}\right) h<C$, and $\beta \leq \bar{\beta}$, where $\beta$ is the root of (A.11), agent 1 delegates decision making to agent 2 who exerts effort, $\beta^{*}=1$, and follows her signal, $\gamma^{*}=0$. Posterior beliefs equal $\widehat{\pi}_{2}\left(X_{1}=1, X_{2}=1\right)=$ $\widehat{\pi}_{2}\left(X_{1}=1, X_{2}=0\right)=\pi_{2}$.

5. If $\lambda>\bar{\lambda}_{C}$ and $\frac{1}{2}\left(\pi_{1}-\pi_{2}\right) h \geq C$, agent 1 exerts effort and follows his signal, whereas agent 2 implements the project without exerting effort, $\beta^{*}=0, \gamma^{*}=1$. The posterior beliefs in case of implementation equals $\widehat{\pi}_{2}\left(X_{1}=1, X_{2}=1\right)=$ $\pi_{2}$, whereas a plausible out-of-equilibrium belief is $\widehat{\pi}_{2}\left(X_{1}=1, X_{2}=0\right)=\left(\frac{1-\pi_{1}}{1-\pi_{1} \pi_{2}}\right) \pi_{2}$.

6. If $\lambda>\bar{\lambda}_{C}$ and $\frac{1}{2}\left(\pi_{1}-\pi_{2}\right) h<C$, agent 1 delegates decision making to agent 2 who exerts effort, $\beta^{*}=1$, and follows her signal, $\gamma^{*}=0$. Posterior beliefs equal $\widehat{\pi}_{2}\left(X_{1}=1, X_{2}=1\right)=\widehat{\pi}_{2}\left(X_{1}=1, X_{2}=0\right)=\pi_{2}$.

Let us interpret these results within the context of our record company example. In case 1 , in which the second expert cares relatively little about her reputation, both experts spend time listening to the demo and giving a sound advice. In the second case, the first expert listens very carefully, whereas the second expert sometimes refrains from listening at all and simply goes along with the first expert's judgment without further ado. The first expert's analysis is sufficiently more likely to be insightful than the second's to merit the effort. There is therefore no reason to delegate 
the decision to the second expert. In the third case, the same type of behaviour results but for a different reason. Now, besides the second expert's moderately strong interest in her reputation, the experts' abilities are very similar. Both constitute reasons to delegate the decision to the second expert. What keeps the first expert from delegating the decision to the second is the fact that although the latter expert sometimes mimics the first expert's judgment she does so with a relatively small likelihood. In the fourth case, this likelihood is substantial. As a result, the expert who is supposed to evaluate the demo first immediately forwards it to the second expert. In both the fifth and the sixth case, the second expert cares considerably about her reputation. Joint decision making is impossible. The first expert then has to decide who should decide on the demo, himself or the second expert? This depends on their relative abilities and the cost of giving a sound advice.

Recall that the economic environment we have assumed in Subsection 2.4 makes sequential decision making optimal. If agent 2 cares little about her reputation, $\lambda<$ $\underline{\lambda}_{C}$, both agents exert effort and follow their private signals. In this case, the total value generated equals the maximally attainable, $\frac{1}{4}\left(1+\pi_{1} \pi_{2}\right) p+\frac{1}{4}\left(\pi_{1}+\pi_{2}\right) h-\frac{3}{2} C$. The cost component $\frac{3}{2} C$ points to one of the advantages of a sequential structure, the fact that agent 2 exerts effort only half of the time. If agent 1 delegates decision making to agent 2 , or if agent 2 does not exert effort with probability one, the project payoff decreases, and the result is inefficient from a project point of view.

Proposition 1 shows that as soon as $\lambda>\underline{\lambda}_{C}$ there is a positive probability that the decision on the project is distorted. An important implication of Lemma 4 is that with endogenous information the project decision is distorted for a wider range of parameters than with exogenous information. Therefore, endogenizing information makes a sequential decision procedure more vulnerable for the adverse consequences of reputational concerns.

\section{Direct Costs Attached to Disagreeing}

In this section, we model the effects of career concerns in a different way ${ }^{15}$. Rather than having the organization's updated belief about the agent's level of competence entering the utility function, we now assume that an agent $i$ suffers a loss $K_{i}$ in case

\footnotetext{
${ }^{15}$ We are grateful to an associate editor for suggesting this approach.
} 
his recommendation does not agree with that of the other agent. An agent's utility function, net of costs of effort becomes:

$$
\begin{aligned}
U_{i}\left(X_{1}=1, X_{2}=1\right) & =p+\mu \\
U_{i}\left(X_{1}=1, X_{2}=0\right) & =-K_{i} \\
U_{i}\left(X_{1}=0\right) & =0
\end{aligned}
$$

We first characterize the second agent's best reply. Define $K_{2}^{*}=-\left(p+\frac{\pi_{1}-\pi_{2}}{1-\pi_{1} \pi_{2}} h\right)-$ $\frac{2}{1-\pi_{1} \pi_{2}} C$.

Lemma 9 (i) Suppose agent 1 has exerted effort and followed his signal. Then agent 2 exerts effort and follows his signal if and only if $K_{2}<K_{2}^{*}$. If this condition fails to hold, agent 2 does not exert effort, but simply accepts the project. (ii) If instead agent 1 has not exerted effort, agent 2 does and follows his signal

To grasp the idea behind Lemma 9, suppose agent 1 has exerted effort. If agent 2 exerts effort, this implies that he is willing to act upon his information. In particular, he is willing to incur a cost $K_{2}$ by rejecting the project in case of a negative assessment. Hence, for agent 2 to exert effort he should not care too much about showing an opinion different from agent 1 , or $K_{2}<K_{2}^{*}$. Of course, if agent 1 does not exert effort, it follows from assumption 2 that agent 2 will.

Now we turn to the equilibria of this model. Define $K_{1}^{*}=-\left(p+\frac{\pi_{2}-\pi_{1}}{1-\pi_{1} \pi_{2}} h\right)-$ $\frac{4}{1-\pi_{1} \pi_{2}} C$.

Proposition 2 Suppose utility functions are defined as in (14). (i) If $K_{2}<K_{2}^{*}$ and $K_{1}<K_{1}^{*}$, then both agents exert effort and follow their respective signals. (ii) If $K_{2}<K_{2}^{*}$ and $K_{1} \geq K_{1}^{*}$, agent 1 forwards the project to agent 2 without analysing it; agent 2 exerts effort and follows his signal. (iii) If $K_{2} \geq K_{2}^{*}$ and $\frac{1}{2}\left(\pi_{1}-\pi_{2}\right) h \geq C$, then agent 1 exerts effort and follows his signal; agent 2 chooses $X_{2}=1$ without analyzing the project. (iv) If $K_{2} \geq K_{2}^{*}$ and $\frac{1}{2}\left(\pi_{1}-\pi_{2}\right) h<C, 1$ forwards the project to 2 without analysing it; agent 2 then exerts effort and follows his signal.

There is an important difference between this model and the one discussed in the previous sections. In the previous model, the degree to which agent 1 cares about his reputation does not influence his decisions. As in equilibrium he cannot influence 
his expected reputation, he bases his decisions on project payoff comparisons only. It can therefore not happen that agent 2 is willing to exert effort and follow his signal, but that agent 1 refrains from exerting effort. In the present model, however, this is exactly what happens in situation (ii). The fact that agent 2 exerts effort and follows his signal, $K_{2}<K_{2}^{*}$, and therefore rejects the project in case of a bad signal, withholds agent 1 from exerting effort if he sufficiently dislikes disagreeing with agent $2, K_{1} \geq K_{1}^{*}$. The other three parts of the proposition are in line with what we found in the previous sections. Parts (i), (iii) and (iv) of Proposition 2 are similar to parts 1, 5 and 6 of Proposition 1, respectively.

\section{Discussion}

We have analysed the implications of endogenising information collection and reputational concerns for the performance of a sequential decision structure. In our model, two agents decide in a sequence whether or not to implement a public project. Each agent is concerned with the outcomes of the project and wants to be reputed as being smart. From the herding literature we know that in a sequential decision structure, reputational concerns may lead to herding. The reason for herding is that agent 2 hurts her reputation by disagreeing with agent 1. Since herding implies that private information is ignored, herding is inefficient from a social point of view.

We show that endogenising information affects the nature of the herding result in two ways. First, given that agent 1 collects information and acts on it, agent 2 does not collect information if she anticipates that she will ignore it when making a decision about the project. Not exerting effort and following agent 1's decision dominates exerting effort and following agent 1's decision.

The second way the herding result is affected is that with endogenous information agent 1 often chooses not to collect information, thereby delegating the decision about the project to agent 2. The reason for this result is a free-rider problem. If agent 2 cares considerably about her reputation, the choice agent 1 faces is between exerting effort himself or delegating the decision to agent 2. Joint decision making is unattainable. Unless agent 1 is much smarter than agent 2, agent 1 prefers a situation in which agent 2 collects information and makes the decision about the project to a situation in which he does the dirty work himself. This second effect of 
endogenising information is important for the answer to the question who effectively makes the decision about the project. With exogenous information and herding, it is the first agent in the sequence who effectively makes the decision about the project. With endogenous information, it is more likely that the second agent makes the decision.

The herding literature shows that reputational concerns may lead to a distortion in the decision process. An important result of our analysis is that with endogenous information an inefficient outcome is more likely to occur. The reason is that even if agent 2 would not herd if she had collected information, reputational concerns increase the cost of acquiring information. The increase in cost stems from the fact that if agent 2 makes a decision in accordance with her information, she may hurt her reputation. Not collecting information and agreeing with agent 1 is always optimal from a reputation point of view.

A direct implication of our results is that in organizations the best man for the job is not always the individual who is most likely to be smart. To prevent agent 1 from delegating the decision about the project to agent 2, an organization may appoint a candidate who is smart with a lower probability than another candidate. The reason is simple. The higher is the probability that the first agent is smart, the stronger is the incentive for the second agent to herd. By hiring an individual who has a relatively low probability of being smart, an organization may prevent the second person from herding. Then, agent 1 has weaker incentives to delegate the decision to the second agent.

In the introduction, we have already mentioned that potentially there are advantages of a sequential decision procedure. We have shown that endogenizing information collection makes sequential decision procedures more vulnerable to adverse consequences of reputational concerns. One interpretation of this result is that in situations in which reputational concerns are important, decisions will not be made by means of a sequential decision procedure. One alternative is delegating the decision to a single agent. When a single agent does not know her type, reputational concerns will not affect her decision on the project (Suurmond, Swank and Visser, 2004). Another alternative is a decision procedure according to which two agents make independent recommendations, so that herding cannot occur.

In this paper, we have assumed that the actual quality of the project will not 
be observed. The implication of this assumption was that the agents' reputations directly follow from their decisions on the project. As only the signals of smart agents are correlated, agreement between agents signals competence, whereas disagreement signals incompetence. At the expense of more algebra, we could have obtained qualitatively similar results in a setting in which (1) the quality of the project is eventually observed; (2) the quality of the project is defined as the sum of two random variables of which one is observed by the agents and the other is not observed; and (3) only the signals of two smart agents are correlated. These assumptions imply that ceteris paribus agreement between the agents still yields a better reputation than disagreement. This holds even if the project turns out the be of low quality. In that case, agreement between the agents makes it relatively more likely that the bad quality is the result of the unobserved random variable. Notice the importance of the existence of the unobserved random variable. Without it, reputations would not depend on agreement or disagreement, but only on whether the agents' decisions are in line with the quality of the project. 


\section{Appendix}

In this section, we first provide some useful mathematical derivations (subsection A.1), we then show that the conditions formulated in the assumptions create the desired environment (A.2), and finally prove the lemmas and the main proposition (A.3). To save space, we write " $g_{i}$ " instead of " $s_{i}=g$ ", and " $b_{i}$ " instead of " $s_{i}=b$ ". Also, instead of writing " $\mu=h "$ we write " $h "$.

\section{A.1 Useful expressions}

It is useful to start with the following (un)conditional probabilities and expected values $\operatorname{Pr}\left(h \mid g_{i}\right)=\pi_{i}+\frac{1}{2}\left(1-\pi_{i}\right)=\frac{1}{2}\left(1+\pi_{i}\right)$. Thus

$$
\begin{aligned}
\operatorname{Pr}\left(h \mid g_{i}\right) & =\frac{1}{2}\left(1+\pi_{i}\right) \\
\operatorname{Pr}\left(-h \mid g_{i}\right) & =\frac{1}{2}\left(1-\pi_{i}\right) \\
\operatorname{Pr}\left(h \mid b_{i}\right) & =\frac{1}{2}\left(1-\pi_{i}\right) \\
\operatorname{Pr}\left(-h \mid b_{i}\right) & =\frac{1}{2}\left(1+\pi_{i}\right)
\end{aligned}
$$

Hence,

$$
E\left(\mu \mid g_{i}\right)=\pi_{i} h=-E\left(\mu \mid b_{i}\right)
$$

In case of two signals,

$$
\begin{aligned}
\operatorname{Pr}\left(g_{i}, b_{j} \mid h\right) & =\frac{1}{2}\left(1-\pi_{i}\right) \frac{1}{2}\left(1+\pi_{j}\right) \\
\operatorname{Pr}\left(g_{i}, b_{j} \mid-h\right) & =\frac{1}{2}\left(1-\pi_{i}\right) \frac{1}{2}\left(1+\pi_{j}\right) \\
\operatorname{Pr}\left(g_{i}, g_{j} \mid h\right) & =\frac{1}{2}\left(1+\pi_{i}\right) \frac{1}{2}\left(1+\pi_{j}\right) \\
\operatorname{Pr}\left(g_{i}, g_{j} \mid-h\right) & =\frac{1}{2}\left(1-\pi_{i}\right) \frac{1}{2}\left(1-\pi_{j}\right)
\end{aligned}
$$

Therefore

$$
\begin{aligned}
\operatorname{Pr}\left(h \mid g_{i}, b_{j}\right) & =\frac{\left(1+\pi_{i}\right)\left(1-\pi_{j}\right)}{2\left(1-\pi_{i} \pi_{j}\right)} \\
\operatorname{Pr}\left(-h \mid g_{i}, b_{j}\right) & =\frac{\left(1-\pi_{i}\right)\left(1+\pi_{j}\right)}{2\left(1-\pi_{i} \pi_{j}\right)}
\end{aligned}
$$


Hence,

$$
E\left(\mu \mid g_{i}, b_{j}\right)=\frac{\pi_{i}-\pi_{j}}{1-\pi_{i} \pi_{j}} h \quad \text { and } \quad E\left(\mu \mid g_{i}, g_{j}\right)=\frac{\pi_{i}+\pi_{j}}{1+\pi_{i} \pi_{j}} h
$$

Also,

$$
\operatorname{Pr}\left(g_{i}, g_{j}\right)=\operatorname{Pr}\left(g_{i}, g_{j} \mid h\right) \operatorname{Pr}(h)+\operatorname{Pr}\left(g_{i}, g_{j} \mid-h\right) \operatorname{Pr}(-h)=\frac{1}{4}\left(1+\pi_{1} \pi_{2}\right)
$$

So

$$
\operatorname{Pr}\left(g_{j} \mid g_{i}\right)=\frac{\operatorname{Pr}\left(g_{i}, g_{j}\right)}{\operatorname{Pr}\left(g_{i}\right)}=\frac{1}{2}\left(1+\pi_{1} \pi_{2}\right)
$$

\section{A.2 The Assumptions}

We now show that the conditions formulated in the assumptions create the desired environment. Assumption 1 guarantees that an individual agent implements the project if he receives signal $g_{i}, p+E\left(\mu \mid g_{i}\right)=p+\pi_{i} h>0$. This follows from equation (A.2). That Assumption 2 ensures that effort is exerted and the signal followed is then immediate. From equation (A.5) it follows that the correct expression has been used in Assumption 3.

Assumption 4 should guarantee that agent 2, conditional on agent 1 having exerted effort and having followed his signal, has an interest in exerting effort. Implementing the project rightaway yields $p+\pi_{1} h$. Exerting effort and following her signal yields $\operatorname{Pr}\left(g_{2} \mid g_{1}\right)\left[p+E\left(\mu \mid g_{1}, g_{2}\right)\right]-C=\frac{1}{2}\left(1+\pi_{1} \pi_{2}\right) p+\frac{1}{2}\left(\pi_{1}+\pi_{2}\right) h-C$ (see equations (A.5) and (A.7)). Thus, effort is exerted if Assumption 4 holds. Finally, assumption 5 should ensure that if agent 2 already exerts effort and follows her signal, it is in the interest of agent 1 to do the same and refer a project to agent 1 only if $s_{1}=g$. The expected payoff for agent 1 if he refrains from exerting effort

equals $\frac{1}{2}\left(p+\pi_{2} h\right)$. If agent 1 participates, the expected payoff for him amounts to $\operatorname{Pr}\left(g_{1}\right)\left[\operatorname{Pr}\left(g_{2} \mid g_{1}\right)\left[p+E\left(\mu \mid g_{1}, g_{2}\right)\right]\right]-C=\frac{1}{4}\left(1+\pi_{1} \pi_{2}\right) p+\frac{1}{4}\left(\pi_{1}+\pi_{2}\right) h-C$. Agent 1 exerts effort if Assumption 5 holds.

\section{A.3 Proofs}

Proof of Lemma 1: Recall that we assume that the strategy of agent 1 is to exert effort and follow his signal. The imputed strategy of agent 2 is to exert effort with probability $\beta$, to implement if $s_{2}=g$, to implement with probability $\gamma$ if $s_{2}=b$, 
and to implement if no effort is exerted. We will show that with this strategy $\widehat{\pi}_{2}\left(X_{1}=1, X_{2}=1\right) \geq \pi_{2}>\widehat{\pi}_{2}\left(X_{1}=1, X_{2}=0\right)$. It is then directly clear that if agent 2 does not exert effort, she prefers project implementation to maintaining the status quo. Project implementation yields both a higher reputation and a positive project payoff by assumption 2 .

Of course, $\widehat{\pi}_{2}\left(X_{1}=1, X_{2}=0\right)=\operatorname{Pr}\left(t_{2}=s m \mid X_{1}=1, X_{2}=0\right)$. Given the imputed strategy, $X_{1}=1$ implies $s_{1}=g$, whereas $X_{2}=0$ implies that agent 2 has exerted effort and received a signal $s_{2}=b$. Thus, $\operatorname{Pr}\left(X_{1}=1, X_{2}=0 \mid t_{2}=s m\right)=$ $\frac{1}{2} \beta\left(1-\pi_{1}\right) \frac{1}{2}$, because if 2 is smart the only way for agents 1 and 2 to have received opposing signals is for agent 1 to be dumb. Similarly, $\operatorname{Pr}\left(X_{1}=1, X_{2}=0 \mid t_{2}=d u\right)=$ $\frac{1}{2} \beta\left(\pi_{1} \frac{1}{2}+\left(1-\pi_{1}\right) \frac{1}{2}\right)=\frac{1}{4} \beta$, as the signal of a dumb agent is uncorrelated with that of the first agent. Using Bayes rule we derive $\widehat{\pi}_{2}\left(X_{1}=1, X_{2}=0\right)=\frac{1-\pi_{1}}{1-\pi_{1} \pi_{2}} \pi_{2}$, which is equation $(2)$. To calculate $\widehat{\pi}_{2}\left(X_{1}=1, X_{2}=1\right)$ recall that with the imputed strategy agent 2 implements a project either because she exerted effort and received a signal $s_{2}=g$; because she exerted effort and she received a signal $s_{2}=b$ and implemented with probability $\gamma$; or because she did not exert effort. Moreover, agent 1 , who passed on the project to agent 2 must have received a signal $s_{1}=g$. Thus,

$$
\begin{aligned}
& \operatorname{Pr}\left(X_{1}=1, X_{2}=1 \mid t_{2}=s m\right) \\
= & \operatorname{Pr}(h) \operatorname{Pr}\left(g_{1} \mid h\right)\left[\beta\left[\operatorname{Pr}\left(g_{2} \mid h, t_{2}=s m\right)+\operatorname{Pr}\left(b_{2} \mid h, t_{2}=s m\right) \gamma\right]+(1-\beta)\right] \\
& +\operatorname{Pr}(-h) \operatorname{Pr}\left(g_{1} \mid-h\right)\left[\beta\left[\operatorname{Pr}\left(g_{2} \mid-h, t_{2}=s m\right)+\operatorname{Pr}\left(b_{2} \mid-h, t_{2}=s m\right) \gamma\right]+(1-\beta)\right] \\
= & \frac{1}{2} \frac{1+\pi_{1}}{2}[\beta+(1-\beta)]+\frac{1}{2} \frac{1-\pi_{1}}{2}[\beta \gamma+(1-\beta)] \\
= & \frac{1}{2} \frac{1+\pi_{1}}{2}+\frac{1}{2} \frac{1-\pi_{1}}{2}[\beta \gamma+(1-\beta)] \\
= & \frac{1}{4}\left(2-\left(1-\pi_{1}\right)(1-\gamma) \beta\right)
\end{aligned}
$$


And

$$
\begin{aligned}
& \operatorname{Pr}\left(X_{1}=1, X_{2}=1 \mid t_{2}=d u\right) \\
= & \operatorname{Pr}(h) \operatorname{Pr}\left(g_{1} \mid h\right)\left[\beta\left[\operatorname{Pr}\left(g_{2} \mid h, t_{2}=d u\right)+\operatorname{Pr}\left(b_{2} \mid h, t_{2}=d u\right) \gamma\right]+(1-\beta)\right] \\
& +\operatorname{Pr}(-h) \operatorname{Pr}\left(g_{1} \mid-h\right)\left[\beta\left[\operatorname{Pr}\left(g_{2} \mid-h, t_{2}=d u\right)+\operatorname{Pr}\left(b_{2} \mid-h, t_{2}=d u\right) \gamma\right]+(1-\beta)\right] \\
= & \frac{1}{2} \frac{1+\pi_{1}}{2}\left[\beta \frac{1}{2}+\beta \frac{1}{2} \gamma+(1-\beta)\right]+\frac{1}{2} \frac{1-\pi_{1}}{2}\left[\beta \frac{1}{2}+\beta \frac{1}{2} \gamma+(1-\beta)\right] \\
= & \frac{1}{4}[\beta(1+\gamma)+2(1-\beta)]
\end{aligned}
$$

From this it immediately follows that $\widehat{\pi}_{2}\left(X_{1}=1, X_{2}=1\right)$ equals the expression in equation $(3), \widehat{\pi}_{2}\left(X_{1}=1, X_{2}=1\right)=\frac{2-\beta(1-\gamma)\left(1-\pi_{1}\right)}{2-\beta(1-\gamma)\left(1-\pi_{1} \pi_{2}\right)} \pi_{2}$.

Proof of Lemma 2: If agent 2 has received a negative signal, whether she prefers to reject or to implement the project is determined by the sign of expression (6). Three cases are possible as far as consistency of agent 2's actions an posterior beliefs are concerned. In case (i), the expression in (6) is negative for $\gamma=0$ substituted in $\widehat{\pi}_{2}\left(X_{1}=1, X_{2}=1\right)-\widehat{\pi}_{2}\left(X_{1}=1, X_{2}=0\right)$. Thus, in this case agent 2 always rejects the project if $s_{2}=b$. Notice that (6) to be negative for $\gamma^{*}=0$ requires that $\lambda<\lambda^{*}$, where $\lambda^{*}$ denotes the value for which (6) equals zero for $\gamma=0$. In case (ii), the expression in (6) is positive for $\gamma=1$ substituted in $\widehat{\pi}_{2}\left(X_{1}=1, X_{2}=1\right)$ $\widehat{\pi}_{2}\left(X_{1}=1, X_{2}=0\right)$. Agent 2 always accepts the project if $s_{2}=b\left(\gamma^{*}=1\right)$. Rather than using her private information, she herds. Case (ii) requires that agent 2 cares sufficiently about her reputation, or $\lambda>\lambda^{* *}$ where $\lambda^{* *}$ is the value of $\lambda$ such that the expression in (6) equals zero for $\gamma=1$. Finally, in case (iii), agent 2 is indifferent between implementation and rejection for some $\gamma^{*} \in[0,1]$. This could be called probabilistic herding. Case (iii) happens for intermediate values of $\lambda: \lambda \in\left[\lambda^{*}, \lambda^{* *}\right]$. Q.E.D.

Proof of Lemma 3: See the text preceding the statement of the lemma. Q.E.D. Proof of Lemma 4: This lemma assumes that agent 1 exerts effort and follows his signal. We first show formally that $\bar{\lambda}_{C}<\lambda^{*}$. Recall that $\lambda^{*}$ is such that expression (6) equals zero for $\gamma=0$. This expression can be rewritten as

$$
\begin{aligned}
& \frac{1}{2}\left(\pi_{1} \pi_{2}-1\right) p+\frac{1}{2}\left(\pi_{2}-\pi_{1}\right) h+ \\
& \frac{1}{2}\left(\pi_{1} \pi_{2}-1\right) \lambda\left[\widehat{\pi}_{2}\left(X_{1}=1, X_{2}=1\right)-\widehat{\pi}_{2}\left(X_{1}=1, X_{2}=0\right)\right]
\end{aligned}
$$


If, for $\gamma=0$ substituted in $\widehat{\pi}_{2}\left(X_{1}=1, X_{2}=1\right)-\widehat{\pi}_{2}\left(X_{1}=1, X_{2}=0\right)$, this expression is positive, agent 2 follows her signal. The payoff to agent 2 if she exerts effort equals

$$
\begin{aligned}
& \frac{1}{2}\left(1+\pi_{1} \pi_{2}\right) p+\frac{1}{2}\left(\pi_{1}+\pi_{2}\right) h+\frac{1}{2}\left(1+\pi_{1} \pi_{2}\right) \lambda \widehat{\pi}_{2}\left(X_{1}=1, X_{2}=1\right)+ \\
& \frac{1}{2}\left(1-\pi_{1} \pi_{2}\right) \lambda \widehat{\pi}_{2}\left(X_{1}=1, X_{2}=0\right)-C
\end{aligned}
$$

whereas the payoff if she does not exert effort amounts to

$$
p+\pi_{1} h+\lambda \widehat{\pi}_{2}\left(X_{1}=1, X_{2}=1\right)
$$

A comparison of these expressions shows that whether agent 2 prefers exerting effort or not is determined by the sign of

$$
\begin{aligned}
& \frac{1}{2}\left(\pi_{1} \pi_{2}-1\right) p+\frac{1}{2}\left(\pi_{2}-\pi_{1}\right) h-C+ \\
& \frac{1}{2}\left(\pi_{1} \pi_{2}-1\right) \lambda\left[\widehat{\pi}_{2}\left(X_{1}=1, X_{2}=1\right)-\widehat{\pi}_{2}\left(X_{1}=1, X_{2}=0\right)\right]
\end{aligned}
$$

If this expression is positive, agent 2 exerts effort. By construction, expression (A.8) equals zero for $\lambda=\lambda^{*}$ and $\gamma=0$. A comparison of (A.8) and (A.11) implies that (A.11) is negative for $\lambda=\lambda^{*}$ and $\gamma=0$, implying that agent 2 does not exert effort, or $\beta^{*}=0$. This also implies that $\bar{\lambda}_{C}<\lambda^{*}$. Let $\underline{\lambda}_{C}$ be the value of $\lambda$ for which (A.11) equals zero after substituting $\beta^{*}=1$ and $\gamma=0$ into $\widehat{\pi}_{2}\left(X_{1}=1, X_{2}=1\right)-$ $\widehat{\pi}_{2}\left(X_{1}=1, X_{2}=0\right)$. If $\lambda<\underline{\lambda}_{C}$, agent 2 always exerts effort and will always follow her signal. Finally, in the third case, we have that $\underline{\lambda}_{C} \leq \lambda \leq \bar{\lambda}_{C}$, and agent 2 exerts effort with probability $\beta^{*} \in[0,1]$, where $\beta^{*}$ is the root of $(\mathrm{A} .11)$ with $\gamma^{*}=1$ : agent 2 will always follow her signal (if she has one), since $\bar{\lambda}_{C}<\lambda^{*}$. $\quad$ Q.E.D.

Proof of Lemma 5: See the text preceding the statement of the lemma. Q.E.D. Proof of Lemma 6: See the text preceding the statement of the lemma. Q.E.D. Proof of Lemma 7: See the text preceding the statement of the lemma. Q.E.D. Proof of Lemma 8: See the text preceding the statement of the lemma. Q.E.D. Proof of Proposition 1: This follows immediately from the lemma 4, and from lemmas 6-8. It may not be directly clear that the constraints in cases (3) and (4) in the proposition are satisfied for some parameter constellations. Suppose $\frac{1}{2}\left(\pi_{1}-\pi_{2}\right) h<C$. Then one can find values $\left\{\underline{\lambda}_{C}, \bar{\lambda}_{C}\right\}$ as long as Assumption 4 
holds (this assumption implies that the top line of (A.11) is positive, while the bottom line is negative for all parameter values). Therefore suppose $\frac{1}{2}\left(\pi_{1}-\pi_{2}\right) h<C$ and $\lambda \in\left[\underline{\lambda}_{C}, \bar{\lambda}_{C}\right]$. Note that the value of $\bar{\beta} \in(0,1)$ is independent of $\lambda$, see (13). Furthermore, $\beta^{*}$ is continuous in $\lambda$, and, if $\lambda \downarrow \underline{\lambda}_{C}$, then $\beta^{*} \uparrow 1$, while if $\lambda \uparrow \bar{\lambda}_{C}$, then $\beta^{*} \downarrow 0$. This guarantees that either case (3) or (4) holds, depending on the parameter values.

Q.E.D.

Proof of Lemma 9: Part (i): First assume that agent 2 has exerted effort. If $s_{2}=g$, he will accept the project as $p+\frac{\pi_{1}+\pi_{2}}{1+\pi_{1} \pi_{2}} h>0>-K_{2}$. If instead $s_{2}=b$, he will reject the project if and only if $K_{2}<-\left(p+\frac{\pi_{1}-\pi_{2}}{1-\pi_{1} \pi_{2}} h\right)$. Now consider agent 2's decision whether to exert effort or not. Assume that once effort has been exerted, he will follow his signal. Exerting effort then yields

$$
\frac{1}{2}\left(1+\pi_{1} \pi_{2}\right)\left(p+\frac{\pi_{1}+\pi_{2}}{1+\pi_{1} \pi_{2}} h\right)+\frac{1}{2}\left(1-\pi_{1} \pi_{2}\right)\left(-K_{2}\right)-C
$$

while not exerting effort will induce him to implement the project as $p+\pi_{1} h>$ $0>-K_{2}$. Hence, he exerts effort if and only if $K_{2}+\frac{2}{1-\pi_{1} \pi_{2}} C<-\left(p+\frac{\pi_{1}-\pi_{2}}{1-\pi_{1} \pi_{2}} h\right)$. Clearly, if this inequality holds, he will follow his signal. Part (ii): This holds by assumption 2 .

Q.E.D.

Proof of Proposition 2: Suppose that agent 2 exerts effort (and therefore follows his signal) if agent 1 exerts effort and follows his signal, $K_{2}<K_{2}^{*}$. Assume agent 1 exerts effort. Will he follow his signal? If $s_{1}=b$, then $X_{1}=0$ best as this yields $-C$, which is more than $\frac{1}{2}\left(1-\pi_{1} \pi_{2}\right)\left(p+\frac{\pi_{2}-\pi_{1}}{1-\pi_{1} \pi_{2}} h\right)+\frac{1}{2}\left(1+\pi_{1} \pi_{2}\right)\left(-K_{1}\right)-C$ (as $p+\frac{\pi_{2}-\pi_{1}}{1-\pi_{1} \pi_{2}} h<0$ and $\left.-K_{1}<0\right)$. If $s_{1}=g$, agent 1 chooses $X_{1}=1$ if and only if

$$
\frac{1}{2}\left(1+\pi_{1} \pi_{2}\right)\left(p+\frac{\pi_{1}+\pi_{2}}{1+\pi_{1} \pi_{2}} h\right)+\frac{1}{2}\left(1-\pi_{1} \pi_{2}\right)\left(-K_{1}\right)>0 .
$$

Now suppose that $K_{2} \geq K_{2}^{*}$, implying that if agent 1 exerts effort and follows his signal, agent 2 does not exert effort and implements the project. If agent 1 exerts effort, it is straightforward to see that he follows his signal.

Will agent 1 exert effort? If he does not exert effort, his payoff equals

$$
\frac{1}{2}\left(p+\pi_{2} h\right)
$$

Assume $K_{2}<K_{2}^{*}$. If (A.12) holds, 1 follows his signal conditional on having one. 
Agent 1 exerts effort if and only if

$$
\frac{1}{4}\left(1+\pi_{1} \pi_{2}\right)\left(p+\frac{\pi_{1}+\pi_{2}}{1+\pi_{1} \pi_{2}} h\right)+\frac{1}{4}\left(1-\pi_{1} \pi_{2}\right)\left(-K_{1}\right)-C>\frac{1}{2}\left(p+\pi_{2} h\right)
$$

or $K_{1}<K_{1}^{*}=-\left(p+\frac{\pi_{2}-\pi_{1}}{1-\pi_{1} \pi_{2}} h\right)-\frac{4}{1-\pi_{1} \pi_{2}} C$. Clearly, if 1 exerts effort, then he follows his signal. This proves part (i). If instead $K_{1} \geq K_{1}^{*}$, agent 1 does not exert effort but forwards the project to agent 2. The latter then exerts effort and follows his signal by assumption 2. This completes the proof of part (ii).

Now assume $K_{2} \geq K_{2}^{*}$. Then agent 2 does not exert effort if agent 1 does. Agent 1 exerts effort himself if $\frac{1}{2}\left(\pi_{1}-\pi_{2}\right) h>C$. If this inequality does not hold, he forwards the project to agent 2 without analysing it. The latter exerts effort and follows his signal by assumption 2. This completes the proof of parts (iii) and (iv). Q.E.D.

\section{References}

Ben-Yasar, Ruth C. and Shmuel I. Nitzan (1997), The Optimal Decision Rule for Fixed-Size Committees in Dichotomous Choice Situations: The General Result, International Economic Review 38 (1), 175-186.

Bikhchandani, Sushil, David Hirschleifer, and Ivo Welch (1998), Learning from the Behavior of Others: Conformity, Fads, and Informational Cascades, Journal of Economic Perspectives 12 (3), 151-170.

Demougin, Dominique (2004), Information Acquisition and Organizational Form, Journal of Institutional and Theoretical Economics 160, 14-17.

Gehrig, Thomas P. (2004), Information Acquisition and Organizational Form, Journal of Institutional and Theoretical Economics 160, 1-14.

Gehrig, Thomas, Pierre Regibeau, and Kate Rockett (2000), Project Evaluation and Organizational Form, Review of Economic Design 5, 177-199.

Keren, Michael and David Levhari (1983), The Internal Organization of the Firm and the Shape of Average Costs, Bell Journal of Economics 14 (2), 474-486.

Koh, Winstion T. H. (1992), Human Fallibility and Sequential Decision Making. 
Hierarchy versus Polyarchy, Journal of Economic Behavior and Organization, 18, $317-345$.

Ladha, Krishna K. (1992), The Condorcet Jury Theorem, Free Speech, and Correlated Votes, American Journal of Political Science, 36, 617-634.

Marschak, Jacob and Roy Radner (1972), Economic Theory of Teams. Yale University Press, New Haven etc.

Ottaviani, Marco and Peter Sorensen (2001) Information Aggregation in Debate: Who Should Speak First?, Journal of Public Economics, 81, 393-421.

Sah, Raaj Kumar and Joseph E. Stiglitz (1986), The Architecture of Economic Systems: Hierarchies and Polyarchies, American Economic Review, 76(4), 716-727.

Sah, Raaj Kumar and Joseph E. Stiglitz (1988), Committees, Hierarchies and Polyarchies, Economic Journal, 98, 451-470.

Scharfstein, David S., and Jeremy C. Stein (1990), Herd Behavior and Investment, American Economic Review 80 (3), 465-479.

Suurmond Guido, Otto H. Swank and B. Visser, (2004), On the Bad Reputation of Reputational Concerns, Journal of Public Economics 88, 2817-2838.

Visser (2000), Organizational Communication Structrure and Performance, Journal of Economic Behavior and Organization, 42, 231-252.

Young, H. Peyton, 1988, Condorcet's Theory of Voting, American Political Science Review, 82,1231-1244. 